Faculdade de Ciências Econômicas UFRGS
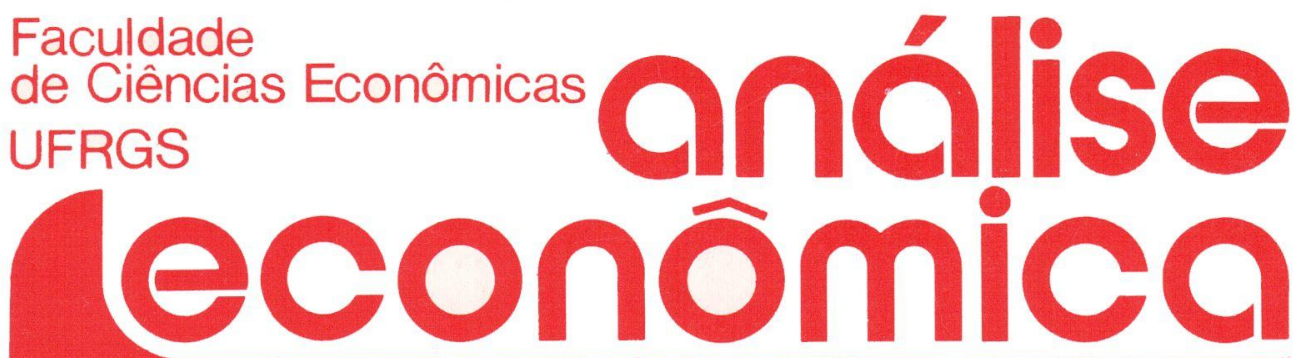

- A LINEAR MOdEL Of BALANCEd GROWTH Joanilio Rodolpho Teixeira Rodrigo Andrés de Souza Penaloza

- TEORIAS ESTRUTURALISTAS DA INFLAÇÃO

Roberto Camps Moraes

- PREÇOS EXTERNOS E EXPÓRTAÇÃO DE MANUFATURADOS Lauro Lobo Burle

- O DESENVOLVIMENTO SUECO Alfredo Marcolin Peringer

- DÉFICIT ENERGÉTICO Fabiano Augusto Nogueira Pinto

- RICARDO E O PROBLEMA SOCIAL Cezar Machado Mello

- UM SABER QUE NÃo SABE: INSTRUMENTO DE PREVISÃO Eleutério F.S. Prado

- SELEÇÃo dE PLANOS DE PRODUÇÃO PARA PEQUENOS PRODUTORES AGRICOLAS Juvir Luiz Mattuella

- PERSPECTIVAS da ECONOMIA do NORDESTE NA DÉCADA DE NOVENTA

Liana Maria da Frota Carleial

- CAIO PRADO JÚNIOR

Pedro Cezar Dutra Fonseca 
UNIVERSIDADE FEDERAL DO RIO GRANDE DO SUL Reitor. Prof. Tuiskon Dick

FACULDADE DE CIÊNCIAS ECONÔMICAS

Diretor: Prof. Walter Meucci Nique

CENTRO DE ESTUDOS E PESQUISAS ECONÔMICAS

Diretor: Reinaldo lgnácio Adams

DEPARTAMENTO DE CIÉNCIAS ECONÔMICAS

Chefe: Prof. Pedro Cezar Dutra Fonseca

CURSO DE PÓS-GRADUAÇÃO EM ECONOMIA

Coordenador: Prof. Nali de Jesus de Souza

CURSO DE PÓS-GRADUAÇĀO EM ECONOMIA RURAL

Coordenador. Prof. Atos Freitas Grawunder

CONSELHO EDITORIAL: Achyles Barcelos da Costa, Aray Miguel Feldens, Atos Freitas Grawunder, Carlos Augusto Crusius, Ernani Hickmann, João Rogério Sanson, Juvir Luiz Mattuella, Maria Imilda da Costa e Silva, Nali de Jesus de Souza, Nuno Renan Lopes de Figueiredo Pinto, Otília Beatriz Kroeff Carrion, Otto Guilherme Konzen, Paulo Alexandre Spohr, Pedro Cezar Dutra Fonseca, Reinaldo Ignacio Adams, Roberto Camps Moraes, Valter José Stülp, Yeda Rorato Crusius, David Garlow (Wharton Econometrics Forecasts Association, E.U.A.), Edgar Augusto Lanzer (UFSC), Eleutério F.S. Prado (USP), Fernando Holanda Barbosa (FGV/RJ), Gustavo Franco (PUC/RJ), Joaquim Pinto de Andrade (UnB), Juan H. Moldau (USP), Werner Baer (Univ. de Illinois, E.U.A.).

COMISSĀO EDITORIAL: Atos Freitas Grawunder, Pedro Cezar Dutra Fonseca, Reinaldo Ignacio Adams e Roberto Camps Moraes.

EDITOR: Nali de Jesus de Souza

SECRETARIA: Maria Ivone de Mello (normalização), Vanete Ricacheski (revisão de textos), Zélide Bregalda (Secretária).

FUNDADOR: Prof. Antônio Carlos Santos Rosa

Os materiais publicados na revista Análise Econômica são de exclusiva responsabilidade dos autores. É permitida a reprodução total ou parcial dos trabalhos, desde que seja citada a fonte.

Aceita-se permuta com revistas congêneres. Aceitam-se, também, livros para divulgação, elaboração de resenhas ou recensōes.

Toda correspondência, material para publicação, assinaturas e permutas devem ser dirigidos ao seguinte destinatário:

PROF. NALI DE JESUS DE SOUZA

Revista Análise Econômica

Av. João Pessoa, 52

90.040 - PORTO ALEGRE (RS), BRASIL

Telefone: (0512) 25-58-44 ramal 33

Fax: (0512) 25-5253 


\section{RELAÇÃO DAS DISSERTAÇÕES DE MESTRADO DOS CURSOS DE ECONOMIA E DE ECONOMIA RURAL DA UFRGS, DEFENDIDAS EM 1990}

a) Economia

ARAÚJO, Nilton Clóvis Machado de. Fatores Locacionais da Agroindústria Alimentar do Rio Grande do Sul. (Orientador: Prof. Nali de Jesus de Souza).

BALBINOTTO NETO, Giácomo. A Indexação Salarial: Teoria e Evidência. (Orientador: Prof. Roberto Camps Moraes).

BRENNER, Geraldo. A Indústria de Calçados do Brasil: Trabalho, Competição e Produtividade. (Orientador. Prof. Carlos Augusto Crusius).

MARCHETTI, Valmor. Incerteza e Risco em Decisão de Investimento Produtivo; Abordagem da Probabilidade. (Orientador: Prof. Carlos Augusto Crusius).

TRICHES, Divanildo. Demanda por Moeda no Brasil e a Causalidade Entre as Variáveis Monetárias e a Taxa de Inflação: 1972-89. (Orientador: Prof. Roberto Camps de Moraes).

VILLAR, Gabriela Isla. Modelos de Distribuição Pessoal de Renda: Uma Revisão. (Orientador: Prof. Carlos Augusto Crusius).

b) Economia Rural

ALCÂNTARA, Maria da Graça de Carvalho. Sistemas de Procuras por Alimentos para a Região Metropolitana de Porto Alegre com Base em Uma Função de Utilidade Quadrática. (Orientador: Prof. Edgar Augusto Lanzer).

CALDART, Wilson Luis. Análise da Demanda Derivada dos Vinhos Produzidos no Rio Grande do Sul. (Orientador: Prof. Edgar Augusto Lanzer). 
FERREIRA, Sonia Maria Brazil: A Análise de Alternativas Agricolas Para Pequenas Propriedades Rurais no Estado de Mato Grosso do Sul. (Orientador. Prof. Otto Guilherme Konzen).

GOMES, Mário Conill. Avaliação Econômica da Conservação do Solo em Microbacias no Município de Marau - RS. (Orientador: Prof. Juvir Luiz Mattuella).

MADAIL, João Carlos Medeiros. Viabilidade Econômica de Novas Técnicas de Produção de Pêssegos a Nível de Persicultores Típicos na Região de Pelotas - RS. (Orientador: Prof. Edgar Augusto Lanzer).

SILVA, Maurício César. Avaliação de Sistemas de Produção de Leite sob Condições de Risco: Região do Vale do Itajaí e Litoral Norte de Santa Catarina. (Orientador: Prof. Otto Guilherme Konzen).

VERRUK, Jaime Elias. O Processo de Industrialização da Fronteira Agricola Brasileira: Estrutura e Dinâmica Industrial do Mato Grosso do Sul. Orientador: Prof. Nali de Jesus de Souza).

ZAPPAROLI, Irene Domenes. Evolução da Estrutura Agrária no Estado do Paraná. (Orientador: Prof. Otto Guilherme Konzen). 